\title{
Habilidades del pensamiento computacional en docentes en formación de la universidad La Gran Colombia
}

Fecha de recepción : 01-03-2021 • Fecha de aceptación: 15-04-2021 • Fecha de publicación: 10-05-2021

\author{
Alvaro Josserand Camargo Pérez ${ }^{1}$ \\ Universidad La Gran Colombia, Colombia \\ alvaroj.camargo@ugc.edu.co \\ https://orcid.org/0000-0002-6268-6840 \\ John Alvaro Munar Ladino² \\ Universidad La Gran Colombia, Colombia \\ john.munar@ugc.edu.co \\ https://orcid.org/0000-0002-1936-8169
}

\section{Resumen}

En la actualidad, uno de los grandes retos de los docentes es atender a los procesos de transformación digital que afronta la sociedad contemporánea. Sin embargo, los profesores no cuentan con las habilidades técnicas ni pedagógicas para desarrollar en sus estudiantes el pensamiento computacional. Una de las principales razones es la falta de programas o currículos formales para la cualificación docente que brinden la posibilidad de desarrollar habilidades como: la descomposición, generalización de patrones, la abstracción y el pensamiento algorítmico.

El objetivo de este proyecto es identificar el nivel de habilidades relacionadas con el pensamiento computacional de los futuros docentes vinculados a la Universidad la Gran Colombia, que quieren ser profesores para luego, establecer estrategias, generar planes de orientación docente, nuevas propuestas curriculares, entre otros. Se utiliza la metodología de enfoque dominante prevaleciendo lo cuantitativo y se utilizó la encuesta como técnica para recopilar información y establecer la relación entre las habilidades y las técnicas de estudio. 
Palabras clave: pensamiento computacional, formación docente, técnicas de estudio, contexto universitario.

\section{Abstract}

Currently, one of the great challenges for teachers is to meet the digital transformation processes faced by contemporary society. However, teachers do not have the technical or pedagogical skills to develop computational thinking in their students. One of the main reasons is the lack of formal programs or curricula for teacher qualification that provide the possibility to develop skills such as: decomposition, pattern generalization, abstraction and algorithmic thinking.

The objective of this project is to identify the level of skills related to computational thinking of future teachers linked to the Universidad la Gran Colombia, who want to become teachers in order to then, establish strategies, generate teaching orientation plans, new curricular proposals, among others. The dominant approach methodology is used, prevailing the quantitative and the survey was used as a technique to collect information and establish the relationship between skills and study techniques.

Keywords: computational thinking, teacher training, study skills, university school context. 


\section{Introducción}

La sociedad actual exige docentes cualificados en sus disciplinas, competentes y capaces de responder a las necesidades propias del entorno como la transformación digital. Sin lugar a duda, el reto de la alfabetización digital ha recaído sobre las instituciones educativas y sus docentes para formar a los ciudadanos más competentes y participes en la construcción de nuevas tecnologías. La apuesta es entonces, desarrollar el pensamiento computacional en niños, niñas y jóvenes para que puedan solucionar problemas, simular experiencias, fomentar la toma de decisiones de forma lógica, ordenada, secuencial, e innovadora que permita crear estrategias para reconocer los factores que puedan afectar a la sociedad.

Como lo afirman Wing (2011) y Valverde et al. (2015), el pensamiento computacional es una competencia básica necesaria para cualquier persona que quiera participar en la sociedad digital de forma inteligente e imaginativa, no se centra en el uso de la tecnología como una solución, sino por el contrario, como un modelo mental para la resolución de problemas, no es sinónimo de capacidad para programar computadores, puesto que se necesita pensar en diferentes niveles de abstracción y es independiente de la tecnología.

Lo anterior, evidencia que el rol docente debe considerar nuevas maneras de relación pedagógica con el estudiante, en el cual no se imponen modelos dados ni tradicionales, sino por el contrario, metodologías alternativas que permitan el desarrollo autónomo, participativo y colaborativo. Las habilidades propias de un docente deben estar centradas en el reconocimiento de los problemas y las estrategias para solucionarlos, evaluar soluciones, recolectar información y analizar datos, entre otras; sin embargo, los currículos y programas de formación y cualificación de futuros docente centran su atención en la alfabetización digital y son pocos los escenarios para desarrollar estas habilidades que exigen el marco de la cuarta revolución industrial. Se puede inferir, como lo menciona Balladares et al. (2016), que el desafío educativo para promover el desarrollo de este pensamiento es fortaleciendo las habilidades del profesorado, dejando de privilegiar la enseñanza sobre el contenido y desarrollando estrategias de enseñanza aprendizaje de manera transversal, tomando en cuenta la realidad de los estudiantes y sus habilidades de pensamiento.

Ahora bien, La Universidad la Gran Colombia cuenta actualmente con 1495 estudiantes adscritos a programas de licenciatura vinculadas a la Facultad de Ciencias de la Educación. La meta general de este proyecto es identificar el nivel de habilidades relacionadas con el pensamiento computacional de los estudiantes que se preparan para ser futuros docentes, para luego establecer estrategias que permitan fortalecer su desarrollo, generar planes de orientación docente, nuevas propuestas curriculares, entre otros.

Para lograr identificar el nivel de competencias relacionadas se realizó un acercamiento conceptual a su definición y sus habilidades, luego se describe el diseño metodológico para su medición con un correspondiente análisis estadístico descriptivo y el último apartado se presenta una discusión con las conclusiones. 


\subsection{Definición}

Actualmente existen varias definiciones acerca del pensamiento computacional, por una parte, Jeannette Wing menciona que es un conjunto de habilidades que "implica la resolución de problemas, el diseño de sistemas y la comprensión de la conducta humana, haciendo uso de los conceptos fundamentales de la informática" (Wing, 2006, p. 33), es decir, que realizando los pasos utilizados en la computación cualquier ser humano puede solucionar problemas de la vida diaria abstrayendo y descomponiendo un problema complejo separándolo en sub-problemas para hacerlo más manejable. La misma autora, en el año 2008 aclara que el pensamiento computacional "incluye los procesos de pensamiento implicados en la formulación de problemas y de sus soluciones, de tal modo que éstos estén representados de una manera que pueda ser abordada efectivamente por un agente procesador de información" (Wing, 2008, p. 3718), lo que significa que cualquier persona, pensando como lo hace un profesional de la computación, puede resolver cualquier problema.

Por otro lado, La International Society for Technology in Education (ISTE) y la Computer Science Teachers Association (CSTA) han brindado una definición operativa basado en un proceso de solución de problemas en el cual es posible utilizar un computador o máquina para organizar, analizar y representar datos; realizar modelos y simulaciones, usar algoritmos para automatizar soluciones, optimizar pasos y recursos, además de generalizar y transferir soluciones a otras situaciones.

Zapotecatl (2018), en su libro "Introducción al Pensamiento Computacional: conceptos básicos para todos", plantea su objetivo como el desarrollo sistémico de habilidades de orden superior como el pensamiento crítico, el razonamiento abstracto y la solución de problemas a través de los conceptos de la informática.

Otra definición es la brindada por Ortega \& Asensio (2018), abordado bajo la relación de dos miradas, la primera desde los procesos de las ciencias de la computación donde toma la descomposición del problema, la automatización, la simulación y el paralelismo y la segunda desde la resolución de problemas con la recopilación de datos, su análisis y representación, abstracción y creación de algoritmos.

\subsection{Habilidades del pensamiento computacional}

De acuerdo con el estudio realizado por el Joint Reserch Center en el año 2016, centro de investigación de la Comisión Europea al servicio de la ciencia y conocimiento, en su informe Desarrollo del pensamiento computacional en la educación obligatoria: implicaciones para la política y la práctica, adopta 6 habilidades para el desarrollo de la solución de un problema: descomposición, abstracción, pensamiento algorítmico, automatización, depuración y generalización.

El estudio mencionado anteriormente, toma las definiciones de Csizmadia (2015) en los cuales se detallan: 
- Abstracción: proceso de hacer más comprensible la información ignorando o depreciando los detalles innecesarios y centrándose en lo importante.

- Pensamiento algorítmico: planteamiento de una solución a través de un plan claro definido de instrucciones paso a paso.

- Automatización: proceso de optimización de trabajo en el que una computadora ejecuta actividades repetitivas de forma más rápida y eficiente que las que realiza un ser humano.

- Descomposición: proceso en que las partes dejan de ser entendidas como un todo y se resuelven, desarrollan y evalúan por separado e independientemente.

- Depuración: aplicación sistemática de análisis y evaluación a través de pruebas, rastreo y pensamiento lógico para predecir, verificar resultados y encontrar errores.

- Generalización: identificación de patrones, similitudes y conexiones para resolver problemas complejos de manera más fácil.

Para los efectos de identificación de habilidades en la población objeto del presente estudio se abordan las cuatro siguientes habilidades del pensamiento computacional: abstracción, pensamiento algorítmico, descomposición y generalización.

La formación de futuros docentes esta permeada por prácticas educativas tradicionales que privilegian los contenidos y la información en vez del desarrollo de habilidades y destrezas para solucionar problemas. Balladares et al. (2016) en su propuesta investigativa titulada "Del pensamiento complejo al pensamiento computacional: retos de la educación contemporánea" centran su atención en encontrar la relación entre estos dos pensamientos con el fin de encontrar coincidencias que permitan fortalecer los procesos de enseñanza - aprendizaje. Los nuevos retos de los educadores van desde entender la nueva lógica de interacción con las tecnologías de la información y la comunicación hasta proponer escenarios y ambientes de aprendizaje donde la innovación, la creatividad, el diseñar actividades dentro y fuera del aula de clase, promuevan el desarrollo de habilidades de sus estudiantes y potencien estrategias metodológicas que involucren aprendizajes significativos para solucionar problemas de la cotidianidad.

Los futuros docentes deben estar a la vanguardia de las necesidades propias de la sociedad, con conocimiento de las tecnologías de la información y la comunicación y la habilidad de adaptarse a los diferentes entornos de aprendizaje. El manejo de las herramientas web, programas y software educativos se vuelven esenciales en la manipulación de los distintos dispositivos, es decir, y en palabras de Valverde et al. (2015), los profesores se deben entender como un profesional que conoce y comprende lenguajes de programación y, que esto, le permite ir más a fondo en la generación de entornos educativos flexibles y trasversales.

Otra propuesta investigativa relacionada es la de Carmona et al., (2017) denominada "Pensamiento computacional en la formación inicial de profesores de matemáticas" en la cual se plantean dos objetivos, el primero evidenciar cómo la configuración de un espacio de formación, 
a partir de las principales características del pensamiento computacional, permitió integrar de manera eficiente y consiente recursos tecnológicos en los diseños curriculares elaborados por los futuros profesores. El segundo, permitió reconocer cómo los futuros profesores (re)significaron la formación en el uso de tecnología dentro la Licenciatura.

Con lo anterior, las facultades de educación deben asumir y reflexionar ante los nuevos desafíos de formación de los futuros docentes en las diferentes disciplinas, vinculando estrategias pedagógicas que desarrollen las habilidades del pensamiento computacional.

\section{Metodología}

La metodología para identificar las habilidades del pensamiento computacional de los estudiantes de las Licenciaturas de la Facultad de Ciencias de la Educación, que se utilizó fue la estrategia de enfoque dominante Hernández et al., (2010), la cual, aunque prevalezca el enfoque cuantitativo y la fuerza la ofrece los datos numéricos, es posible interpretar y analizar cualitativamente a través de juicios y valoraciones. El diseño es de tipo no experimental y transaccional, puesto que no se manipulan variables y se observa el fenómeno en su forma natural y solo se recolectan los datos por una única vez.

La técnica usada para la recolección de datos es la encuesta Grasso (2006), la cual permite indagar subjetividades y actitudes a un número alto de participantes; admite la búsqueda sistémica de información y recoge las actitudes de conductas no susceptibles de observación directa. El instrumento de recolección es el cuestionario online, aplicado a través de internet debido a la situación de pandemia y a la imposibilidad de realizar actividades presenciales con los participantes. El instrumento fue elaborado por los investigadores y fue aplicado de forma anónima.

Conocer la situación actual de las habilidades de los futuros docentes de la Facultad de Educación relacionadas con el pensamiento computacional es necesaria para establecer estrategias formativas, en especial cuando no existe dentro de las mallas curriculares de los programas adscritos a la facultad espacios académicos relacionados, ni otras estrategias formativas que ayuden a determinar el estado presente de esas habilidades. En otros estudios, como los de Olabe et al., (2015) y Barrera \& Montaño (2015) se han determinado el nivel de habilidades a través de la programación de juegos interactivos, en especial bajo el lenguaje de SCRATCH, que permite la generación de contenidos basado en bloques y el desarrollo de actividades que se triangulan con las habilidades del pensamiento computacional.

Al carecer de lo anteriormente mencionado, fue necesario buscar algún ejercicio rutinario y en contexto de los estudiantes de la facultad, que tuvieran una aproximación con estas habilidades y establecer una relación. Las técnicas de estudio fueron el punto de encuentro, como lo menciona Enríquez et al., (2015), son una práctica adquirida que al ser recurrente se adquieren habilidades que conllevan estrategias de observación, percepción, organización, comparación, interpretación, análisis, síntesis, generalización, entre otros.

Ahora bien, el cuestionario se estructuró en dos secciones, la primera constó de tres preguntas de tipo identificación que caracterizaron la población con respecto a género, edad y programa de 
la facultad al que pertenecen. La segunda sección indagó en cuatro preguntas, sobre las actitudes relacionadas con las habilidades del pensamiento computacional de los estudiantes al momento de utilizar técnicas de estudio para la preparación de un examen. Las preguntas de este apartado correspondieron a:

- Pregunta 4: relaciona las acciones de los participantes con la habilidad de descomposición, por lo tanto, está orientada hacia el reconocimiento de cómo implementan procesos de separación de algo complejo en partes más pequeñas, para ser abordadas de forma independiente. El contexto de la pregunta se conecta con el momento en que los participantes estudian para una prueba final, planteada de la siguiente forma: Cuando usted estudia para un examen, ¿divide los contenidos, categoriza los temas relevantes y busca las relaciones entre ellos?

- Pregunta 5: aborda la habilidad de generalización de patrones que busca similitudes, lazos y relaciones de particularidades para la resolución de un problema. Para el diseño de esta pregunta se establece una relación con las formas de estudio de los participantes para una prueba final y su habilidad para generalizar, la pregunta es: Cuando usted estudia para un examen, ¿utiliza estrategias de estudio para establecer similitudes y relaciones entre los contenidos y las actividades de la clase?

- Pregunta 6: indaga sobre la habilidad de la abstracción, proceso en el cual los participantes pueden comprender más fácilmente una situación eliminando o reduciendo datos no necesarios. La situación planteada se relaciona con la toma de apuntes en clase que depende tanto del ritmo de escritura del participante, como de su capacidad para discriminar y sintetizar la información relevante. La pregunta planteada es: Cuando usted estudia para un examen, ¿realiza resúmenes de los temas vistos?

- La última pregunta se relaciona con la habilidad del pensamiento algorítmico, la cual define la capacidad de resolución de problemas a través de instrucciones, reglas o pasos ordenados. Para este caso se busca reconocer si los participantes realizan representaciones ordenadas y secuenciales que faciliten comprender, entender o apreciar mejor los contenidos. La pregunta planteada es: Cuando usted estudia para un examen, ¿diseña esquemas ordenados y secuenciales que faciliten el aprendizaje?

Teniendo en cuenta la pretensión de la investigación se utilizó la escala Likert, este método, tal como lo menciona Hernández et al., (2010), consiste en un grupo de ítems expuestos a manera de juicios, mediante los cuales se considera la reacción de los participantes objeto de estudio. Se plantearon 4 ítems valorados entre 1 y 4, los valores 1 y 2 muestran una actitud desfavorable, mientras que los valores 3 y 4 actitudes favorables o positivas.

La Universidad La Gran Colombia cuenta con cinco facultades, para esta investigación se trabajó únicamente con la Facultad de Ciencias de la Educación, encargada de brindar estudios superiores para formar profesionales especializados en docencia, didáctica y pedagogía. Se excluyen las demás facultades por la idea de generar posteriormente propuestas curriculares relacionadas entre el pensamiento computacional y la formación de futuros profesores. Para el primer semestre académico del año 2020, la facultad contó con 1.495 estudiantes vinculados a los 6 programas de 
Licenciaturas. La muestra fue de 381 estudiantes distribuidos como se evidencia en la Tabla 1.

Tabla 1.

Población y muestra estudiantil primer periodo 2020 Facultad de Ciencias de la Educación

\begin{tabular}{|l|c|c|}
\hline Programa & Población & Muestra \\
\hline Licenciatura en Educación Infantil & 21 & 18 \\
\hline $\begin{array}{l}\text { Licenciatura en Matemáticas y Tecnologías de la } \\
\text { Información }\end{array}$ & 10 & 3 \\
\hline Licenciatura en Lenguas Modernas & 838 & 163 \\
\hline Licenciatura en Filosofía & 60 & 24 \\
\hline Licenciatura en Humanidades y Lengua Castellana & 187 & 59 \\
\hline Licenciatura en Ciencias Sociales & 379 & 114 \\
\hline TOTAL & 1495 & 381 \\
\hline
\end{tabular}

Fuente: elaboración propia

\section{Resultados}

De los 381 participantes, el 42,78\% pertenecen al programa de Licenciatura en Lenguas Modernas con énfasis en inglés, el 29,92\% a la Licenciatura en Ciencias Sociales, el 15,49\% a la Licenciatura en Humanidades y Lengua Castellana, el 6,3\% a la Licenciatura Educación Infantil, el 4,72\% a la Licenciatura en Filosofía y por último el $0,79 \%$ a la Licenciatura en Matemáticas, el número de participantes por programa se encuentra en la Figura 1.

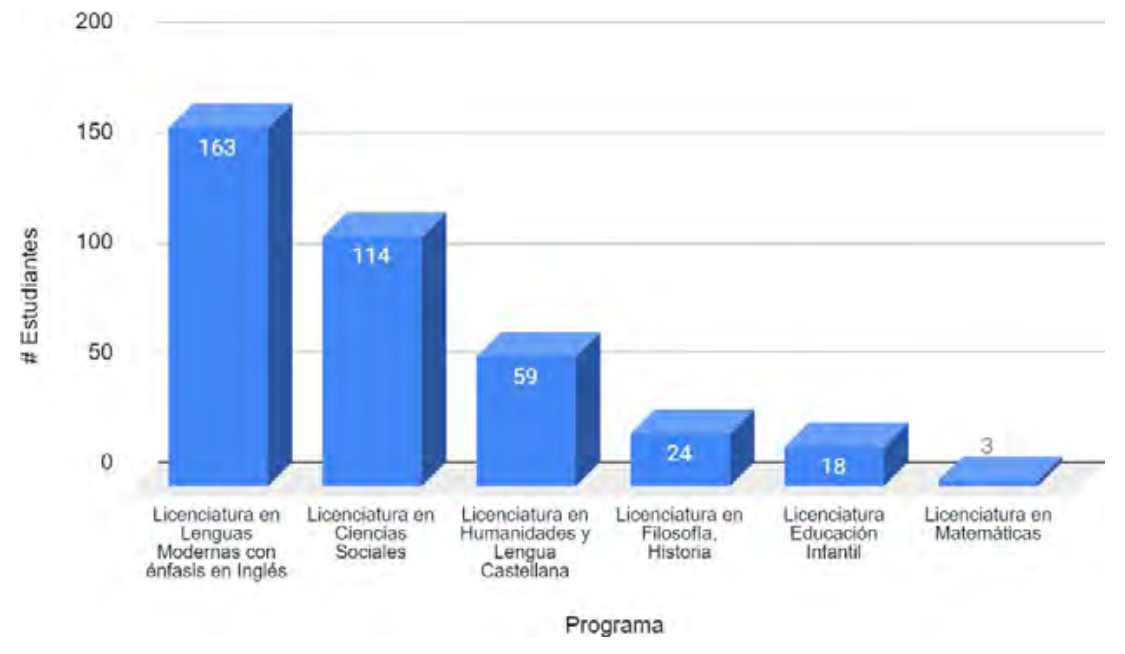

Figura 1. Número de estudiantes por programa

Fuente: elaboración propia

Con respecto al género el 55,9 \% que contestaron la encuesta fueron mujeres y correspondieron 
a 213 participantes, mientras que el 44,1\% fueron hombres con una equivalencia de 168 participantes. Para la categoría de edad, 193 participantes se encuentran en el rango entre los 18 y 22 años, 102 entre los 23 y 27 años, 34 entre los 28 y 32 igual que los menores de 18 años, 13 participantes entre los 33 y 37 años, 4 entre los 38 y 42 años y un participante entre los 43 y 47 años. El porcentaje de participantes por rango de edad se encuentra en la Figura 2.

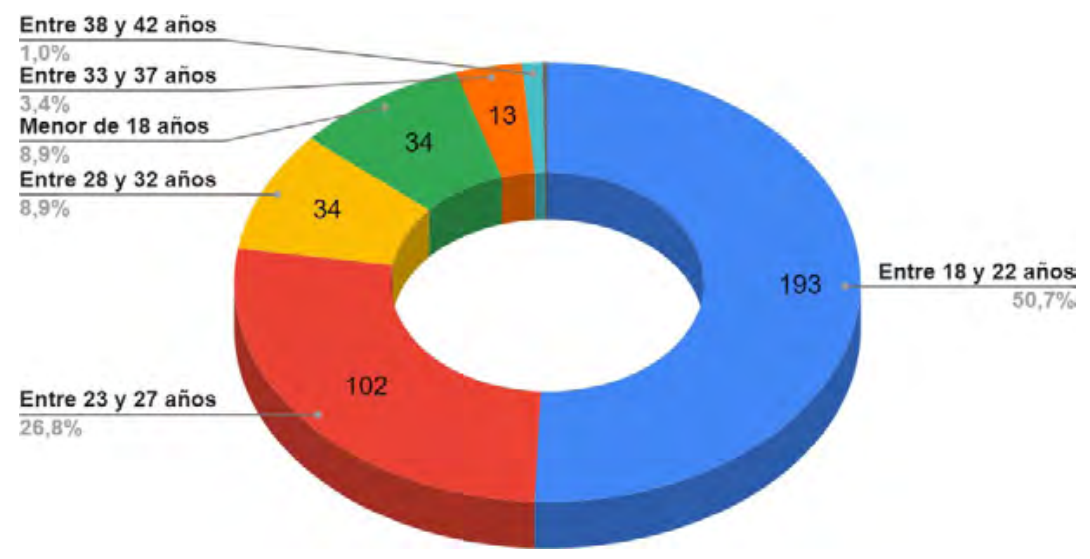

Figura 2. Porcentaje de Estudiantes por rango de edad.

Fuente: elaboración propia

Ante las inquietudes relacionadas con las habilidades del pensamiento computacional: descomposición, generalización de patrones, abstracción y pensamiento algorítmico se organizan los datos de tal manera que a cada actitud comportamental se le asigna una escala de categoría de tipo de frecuencia. Además, por cada pregunta se presenta la media, los puntos de escala Likert y su distribución porcentual de estudiantes por ítem.

Para cada ítem de respuesta se asignó entre 1 y 4 puntos. Para el presente análisis, se muestra por pregunta el consolidado de puntos de los 381 estudiantes encuestados dando como rango por pregunta entre 381 a 1.524 puntos. En este análisis de datos, la interpretación que se toma de la sumatoria de puntos de escala Likert se presenta en la Tabla 2.

Tabla 2.

Escala de puntos según actitudes

\begin{tabular}{|l|l|}
\hline ACTITUD & PUNTOS \\
\hline Desfavorable & Entre 381 y 952 puntos \\
\hline Favorable & Entre 953 a 1524 puntos \\
\hline
\end{tabular}

Fuente: elaboración propia

En la Tabla 3 se sintetiza los datos recogidos en la aplicación del cuestionario para cada una de las habilidades. 
Tabla 3.

Resumen de los datos por habilidad.

\begin{tabular}{|c|c|c|c|c|c|c|}
\hline Habilidad & Ítem & $\begin{array}{l}\text { Frecuencia } \\
\text { Absoluta Fi }\end{array}$ & Porcentaje & $\begin{array}{l}\text { Porcentaje } \\
\text { acumulado }\end{array}$ & Suma $\Sigma$ & Media \\
\hline Descomposición & Siempre & 199 & $52,20 \%$ & $52,20 \%$ & \multirow{4}{*}{1226} & \multirow{4}{*}{3,21} \\
\hline & $\begin{array}{l}\text { Algunas } \\
\text { veces }\end{array}$ & 116 & $30,40 \%$ & $82,70 \%$ & & \\
\hline & $\begin{array}{l}\text { Pocas } \\
\text { veces }\end{array}$ & 16 & $4,20 \%$ & $86,90 \%$ & & \\
\hline & Nunca & 50 & $13,10 \%$ & $100,00 \%$ & & \\
\hline \multirow{4}{*}{$\begin{array}{l}\text { Generalización } \\
\text { de Patrones }\end{array}$} & Siempre & 145 & $38,10 \%$ & $38,10 \%$ & \multirow{4}{*}{1170} & \multirow{4}{*}{3,07} \\
\hline & $\begin{array}{l}\text { Algunas } \\
\text { veces }\end{array}$ & 155 & $40,70 \%$ & $78,70 \%$ & & \\
\hline & $\begin{array}{l}\text { Pocas } \\
\text { veces }\end{array}$ & 44 & $11,50 \%$ & $90,30 \%$ & & \\
\hline & Nunca & 37 & $9,70 \%$ & $100,00 \%$ & & \\
\hline \multirow[t]{4}{*}{ Abstracción } & Siempre & 136 & $35,70 \%$ & $35,70 \%$ & \multirow{4}{*}{1084} & \multirow{4}{*}{2,84} \\
\hline & $\begin{array}{l}\text { Algunas } \\
\text { veces }\end{array}$ & 139 & $36,50 \%$ & $72,20 \%$ & & \\
\hline & $\begin{array}{l}\text { Pocas } \\
\text { veces }\end{array}$ & 17 & $4,50 \%$ & $76,60 \%$ & & \\
\hline & Nunca & 89 & $23,40 \%$ & $100,00 \%$ & & \\
\hline \multirow{4}{*}{$\begin{array}{l}\text { Pensamiento } \\
\text { Algorítmico }\end{array}$} & Siempre & 38 & $10,00 \%$ & $10,00 \%$ & \multirow{4}{*}{802} & \multirow{4}{*}{2,1} \\
\hline & $\begin{array}{l}\text { Algunas } \\
\text { veces }\end{array}$ & 118 & $31,00 \%$ & $40,90 \%$ & & \\
\hline & $\begin{array}{l}\text { Pocas } \\
\text { veces }\end{array}$ & 71 & $18,60 \%$ & $59,60 \%$ & & \\
\hline & Nunca & 154 & $40,40 \%$ & $100,00 \%$ & & \\
\hline
\end{tabular}

Fuente: elaboración propia

Las habilidades relacionadas con descomposición, generalización de patrones, abstracción y pensamiento algorítmico se representan de acuerdo a la sumatoria de puntos de escala Likert entre 381 a 1524 puntos. Evidenciando las 3 primeras con actitudes favorables, mientras el pensamiento algorítmico con actitud desfavorable, como se evidencia en la Figura 3. 


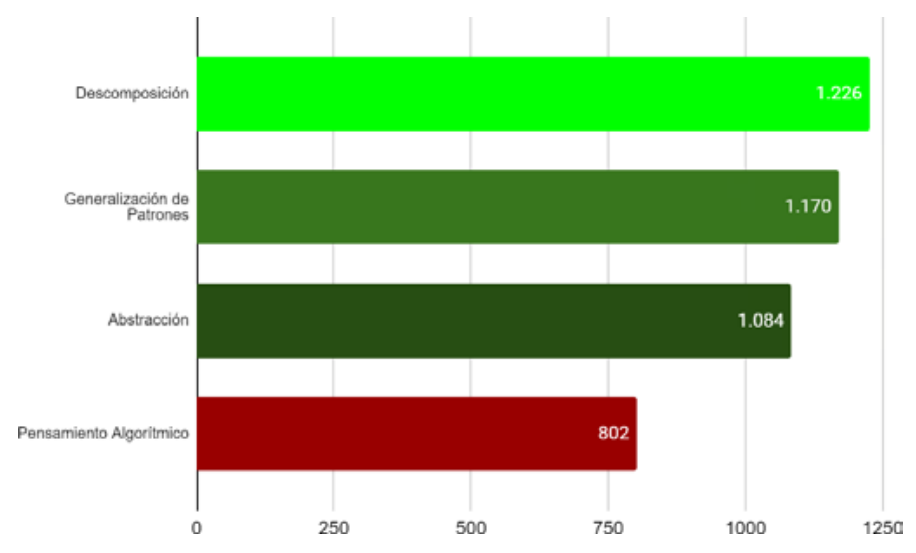

Figura 3. Suma de puntos por habilidad.

Fuente: elaboración propia

A partir de los datos encontrados, se evidencia que existe una relación entre las habilidades del pensamiento computacional con las técnicas de estudio de los estudiantes, entendidas de acuerdo con Ayma (1996) como un conjunto de estrategias y procedimientos que permiten mejorar el rendimiento académico vinculado con procesos de aprendizaje. Se destaca como el uso de la abstracción, descomposición, la generalización y el pensamiento algorítmico pueden presentarse en los hábitos de estudio de los estudiantes de manera automática.

Ante la habilidad relacionada con descomposición, los estudiantes encuestados presentaron una media 3,21 y una sumatoria de puntos de 1.226/1524. Se encontró que el $82,7 \%$ de los estudiantes al momento de estudiar para una evaluación presentan actitudes favorables al separar y categorizan los contenidos, quiere decir que presentan esa capacidad de análisis para deconstruir un todo en contenidos más simples para facilitar su comprensión. La descomposición se evidencia notablemente en las técnicas de estudio de elaboración y organización como lo mencionan Enríquez et al. (2015) al separar tareas simples y tareas complejas, la primera a través de asociación de pares como vocabularios y listas, y la segunda por medio de actividades más elaboradas como parafrasear, resumir, tomar notas, entre otras. Por otra parte, cada tarea requiere su comprensión para poder clasificar ordenar y agrupar.

Ahora bien, para la habilidad de generalizar la media correspondió a 2,84 y la sumatoria de puntos sobre 1170/1524. El 78,8\% de los estudiantes encuestados manifestaron una actitud positiva al momento de utilizar estrategias para establecer similitudes y relaciones entre contenidos y actividades de clase. Al respecto, Zapata (2015) esta actividad exige la capacidad de distinguir elementos comunes y dar pautas en situaciones distintas, se aplica en la computación y otras disciplinas ya que genera "buenas prácticas" y da referencias para solucionar nuevos problemas pues optimizan el trabajo intelectual y vuelve más eficaz el trabajo empleado. Para encontrar situaciones de aprendizaje que brinden semejanzas y relaciones entre contenidos y actividades curriculares, Enríquez et al. (2015) menciona que es posible agrupar técnicas de estudio como son la comparación, que permite establecer diferencias y semejanzas entre dos o más datos, la deducción que establece juicios generales y particulares a partir de hechos, y por último la comprensión que da entendimiento y significado a los datos para generar asociaciones. 
La tercera habilidad es asociada a la abstracción, la media correspondió a 3,07 y la sumatoria de puntos sobre 1084/1524. Los resultados mostraron que el $72,2 \%$ de los estudiantes encuestados presentaron una actitud favorable al momento de discriminar y sintetizar la información importante. El resumen es en efecto una técnica de estudio que permite realizar una relación con la habilidad de abstraer, ya que al transferir de un documento a otro las ideas principales, requiere de acuerdo con Enríquez et al. (2015) un reconocimiento de temas, una identificación de estructuras, resaltar lo sustancial, organización de ideas, permite generar conceptos a partir de la extracción de propiedades complejas y enfoca la atención en lo esencial para resolver un problema

La última habilidad es el pensamiento algorítmico, la media correspondió a 2,01 y la sumatoria de puntos sobre $802 / 1524$ evidenciando que el $59 \%$ de los estudiantes encuestados manifestaran una actitud desfavorable al diseñar esquemas ordenados y secuenciales que faciliten su proceso de aprendizaje. Este tipo de método de estudio requiere según Enríquez et al. (2015) representar estructuras jerárquicas y de flujo que destaquen los procesos y actividades por pasos o ciclos.

\section{Conclusiones}

A pesar que son pocos los currículos o programas de cualificación docente formales que brinden espacios de desarrollo de las habilidades relacionados con el pensamiento computacional, es posible realizar un acercamiento a los docentes en formación con ciertas habilidades adquiridas al momento de utilizar las técnicas de estudio. Fortalecer el pensamiento computacional en estos estudiantes es una apuesta a considerar por las facultades de educación universitaria dentro de sus currículos, puesto que este se entenderá como una habilidad fundamental que debe desarrollarse desde la escuela respondiendo a las nuevas necesidades del siglo XXI.

La experiencia reveló un número significativo de estudiantes con actitudes favorables ante las habilidades relacionadas con la abstracción, la descomposición y la generalización, por el contrario, ante el pensamiento algorítmico los resultados fueron actitudes desfavorables, lo anterior se debe posiblemente a la exigencia y dedicación necesaria para implementar estos procedimientos, puesto que requiere ordenar, jerarquizar y sintetizar ideas. Pensar algorítmicamente necesita seguir instrucciones paso a paso, tener presteza y persistencia para lograr resolver problemas complejos. La programación es sin duda un elemento esencial para el desarrollo del pensamiento computacional, requiere a través de la lógica, el razonamiento y la capacidad de dar instrucciones la posibilidad de solucionar problemas.

La dificultad de incluir el desarrollo del pensamiento computacional en las primeras edades de formación, se debe al escaso número de profesionales de la educación preparados para enseñarlo, es por eso, que resulta necesario plantear estrategias curriculares dentro de los planes de estudio de los programas universitarios relacionados con la formación de docentes. 


\section{Referencias}

Ayma, G. (1996). Curso: Enseñanza de las Ciencias: Un enfoque constructivista. Instituto de Física y Facultad de Educación. Universidad de Sao Paulo.

Barrera, C, R. \& Montaño, R. E. (2015). Desarrollo del pensamiento computacional con Scratch. In J. Sánchez (Presidente), Nuevas ideas en informática educativa. Simposio llevado a cabo en el XX Congreso internacional de Informática Educativa, Santiago, Chile.

Balladares, B., J., Avilés, S. R., \& Pérez, N., O., P. (2016). Del pensamiento complejo al pensamiento computacional: retos para la educación contemporánea. Sophia: Colección de Filosofía de la Educación, (21), 143-159. https://doi.org/10.17163/soph.n21.2016.06

Carmona M, J. A., Cardona, M., y Villa-Ochoa, J. A. (2017). Pensamiento computacional en la formación inicial de profesores de matemáticas. Informe técnico de proyección de innovación. Medellín, Colombia.

Csizmadia, A., Curzon, P., Dorling, M., Humphreys, S., Ng, T., Selby, C. y Woollard, J. (2015) Pensamiento computacional Una guía para profesores. Computación en la escuela.

CSTA \& ISTE. (2011). Computational Thinking Leadership Toolkit, First edition. Computer Science Teachers Association (CSTA) y International Society for Technology in Education (ISTE). https://goo.gl/syFwSF.

Enríquez, M. F., \& Fajardo, M. \& Garzón F. (2015). Una Revisión General a los Hábitos y Técnicas de Estudio en el Ámbito Universitario. Psicogente, 18(33),166-187. https://doi.org/10.17081/psico.18.33.64

Grasso, L. (2006). Encuestas. Elementos para su diseño y análisis. Córdoba: Encuentro Grupo Editor, 27-56.

Hernández, R., Fernández, C. C. y Baptista, L. P. (2010). Metodología de la Investigación. México: McGraw Hill. Quinta Edición.

Olabe, X. B., Basogain, M. Á. O., \& Basogain, J. C. O. (2015). Pensamiento Computacional a través de la Programación: Paradigma de Aprendizaje. Revista de educación a distancia (RED), (46).

Ortega R, B., \& Asensio B, M. M. (2018). Robótica DIY: pensamiento computacional para mejorar la resolución de problemas.

Valverde B, J., Fernández, M. R., \& Garrido A, M. del C. (2015). El pensamiento computacional y las nuevas ecologías del aprendizaje. Revista De Educación a Distancia (RED), (46). https://doi. org/10.6018/red/46/3

Wing, J. M. (2006). Computational thinking. Communications of the ACM, 49(3), 33-35. https://doi. 


\section{org/10.1145/1118178.1118215}

Wing, J. M. (2008). Computational thinking and thinking about computing. Philosophical Transactions. Series

A, Mathematical, Physical, and Engineering Sciences, 366(1881), 3717- 3725. https://doi.org/10.1098/ rsta.2008.0118

Wing, J. M. (2011). Computational thinking. En 2011 IEEE Symposium on Visual Languages and Human-Centric Computing (VL/HCC 2011) (pp.3-3). IEEE. https://doi.org/10.1109/VLHCC.2011.6070404

Zapata, R. M. (2015). Pensamiento computacional: Una nueva alfabetización digital. Revista de Educación a distancia, (46).

Zapotecatl López, J. L. (2018). Introducción al pensamiento computacional: conceptos básicos para todos. 
Copyright (c) 2021 Alvaro Josserand Camargo Pérez y John Alvaro Munar Ladino

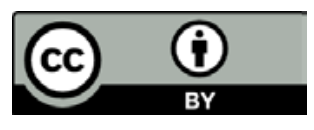

Este texto está protegido bajo una licencia internacional Creative Commons 4.0.

Usted es libre para Compartir-copiar y redistribuir el material en cualquier medio o formato - y Adaptar el documento - remezclar, transformar y crear a partir del material-para cualquier propósito, incluso para fines comerciales, siempre que cumpla las condiciones de Atribución. Usted debe dar crédito a la obra original de manera adecuada, proporcionar un enlace a la licencia, e indicar si se han realizado cambios. Puede hacerlo en cualquier forma razonable, pero no de forma tal que sugiera que tiene el apoyo del licenciante o lo recibe por el uso que hace de la obra.

$\underline{\text { Resumen de licencia - Texto completo de la licencia }}$ 